\title{
YouTube as a Source of Information on Epidural Steroid Injection
}

\author{
Min Cheol Chang' \\ Donghwi Park (D) ${ }^{2}$ \\ 'Department of Physical Medicine and \\ Rehabilitation, College of Medicine, \\ Yeungnam University, Daegu, Republic of \\ Korea; ${ }^{2}$ Department of Physical Medicine \\ and Rehabilitation, Ulsan University \\ Hospital, University of Ulsan College of \\ Medicine, Ulsan, Republic of Korea
}

\begin{abstract}
Objective: With the advance of the internet, social media platforms have become a major source of medical information. We assessed the reliability, quality, and usefulness of the
\end{abstract} most-viewed YouTube videos of epidural steroid injection (ESI).

Methods: A search was conducted on YouTube on February 13, 2020, using the keywords "epidural injection," "epidural steroid injection," "epidural transforaminal injection," and "epidural transforaminal steroid injection." The top 50 most-viewed videos were assessed with a modified DISCERN scale (mDISCERN) and a Global Quality Scale (GQS). Further, the usefulness of information in each video was evaluated.

Results: Only $22 \%$ of videos contained information with high reliability, and these were produced by hospitals or physicians. None of the videos provided by media organizations and patients were reliable. As for information quality, only $34 \%$ were moderate to excellent quality. Even of the videos produced by hospitals or physicians, approximately half were of generally poor or poor quality. Regarding the usefulness of information, although $76 \%$ were assessed to contain useful information, $8 \%$ had misleading information. Particularly, four of these videos contained misleading information, and three were provided by patients who experienced ESI.

Conclusion: YouTube is a platform where medical information is actively shared and widespread. Here, we found that the reliability and quality of videos were low even when these were produced by hospitals or physicians. Further, the quality tended to be much lower when it was provided by media organizations or patients. Future efforts by physicians and professional societies to improve the reliability and quality of medical content are necessary. Keywords: epidural steroid injection, back pain, sciatica, online media, quality, social media

\section{Introduction}

Lower back pain (LBP) and sciatica are common complaints of patients and affect a major proportion of the population. ${ }^{1}$ Because LBP and sciatica cause disability and decrease quality of life, appropriate management of those symptoms is important. ${ }^{2}$ For management of LBP and sciatica, various conservative treatments, including oral pain medication, physical therapy, exercise, and interventions, are being applied. ${ }^{3-5}$ Of these treatment modalities, epidural steroid injection (ESI) has been applied widely in patients with LBP or sciatica. ${ }^{6}$ The anti-inflammatory property of steroids reduces inflammation around the sinuvertebral nerve and spinal nerve roots. ${ }^{7}$ Additionally, steroids have a function to inhibit neural transmission within the nociceptive C-fibers. ${ }^{8}$ These actions of steroids can reduce pain following ESI.

Recently, ESI has become one of the most frequently applied procedures in pain clinics; therefore, patients' interest in this procedure is increasing and they
Correspondence: Donghwi Park Department of Physical Medicine and Rehabilitation, Ulsan University Hospital, University of Ulsan College of Medicine, 877, Bangeojinsunghwando-ro, Dong-gu, Ulsan, 44033, Republic of Korea

Tel +82-52-250-7222

Fax +82-52-250-7228

Email bdome@hanmail.net 
require accurate information, such as indication, effect, and adverse effect, on the procedure. ${ }^{6}$ With the advance of the Internet, people can easily obtain health information online, making it the most prominent source of health information. Patients also seek information on medical procedures in social media, in which experiences of real patients and opinions of several medical experts are provided. However, online multimedia platforms can contain inaccurate medical information, which can hinder patients from making informed decisions on the treatment of disorders. ${ }^{9}$ YouTube is the most popular and largest media-sharing platform online, and it is considered an important platform for dissemination of medical information. ${ }^{10}$ Therefore, in the current study, we evaluated the reliability, quality, and usefulness of the most-viewed YouTube videos about ESI.

\section{Methods}

\section{Video Selection}

This cross-sectional study conducted a search on https:// www.youtube.com/on February 13, 2020, using the keywords "epidural injection," "epidural steroid injection," "epidural transforaminal injection," and "epidural transforaminal steroid injection." Inclusion criteria for videos were 1) primary content related to ESI and 2) English language. Exclusion criteria were 1) duplicated videos and 2) no audio. The 50 most-viewed videos satisfying the above criteria were included in the review. Ethics committee approval was not required as this study did not include any human participants and the videos were publicly accessible.

\section{Data Extraction}

We extracted data from each video. Extracted data included title, production source, duration on YouTube, video length, and total number of views, likes, dislikes, and subscribers. The video production source was categorized into 1) hospitals or physicians; 2) patients; or 3) media organizations.

\section{Assessment of Reliability, Quality, and Usefulness}

The reliability of video content was assessed using the modified DISCERN scale (mDISCERN), which was adapted from the original DISCERN for the assessment of written health information by Charnock et al. ${ }^{11}$ The mDISCERN scale includes five questions: 1) are the aims clear and achieved; 2) are reliable sources of information used; 3) is the information presented balanced and unbiased; 4) are additional sources of information listed for patient reference; and 5) are areas of uncertainty mentioned. A higher score in mDISCERN indicate greater reliability. When a mDISCERN score is 3 or greater, the information is highly reliable. ${ }^{10}$

The Global Quality Scale (GQS) was used for assessing the quality of video content. ${ }^{12}$ This evaluation tool was originally developed to evaluate website resources and assess the flow and ease of use of the information available. In GQS, the information is classified as follows: 1) poor quality, poor flow, and most information is missing, so that it is not helpful for patients; 2) generally poor, with some information given but of limited use to patients; 3) moderate quality and some important information is adequately discussed; 4) good quality, good flow, and most relevant information is covered, making it useful for patients; and 5) excellent quality and excellent flow, making it very useful for patients. A higher GQS score means greater quality information.

In addition, each video was classified as either useful, misleading, or neither useful nor misleading. ${ }^{10}$ The criteria for useful videos included 1) at least one correct scientific statement about the definition of ESI; 2) at least one correct statement of pain-reducing mechanism of ESI; or 3 ) at least one accurate clinical statement about the indications, adverse effects, benefits, and potential outcomes of ESI. The criteria for misleading videos included 1) at least one inaccurate scientific statement about the definition of ESO; 2) at least one inaccurate statement about the pain-reducing mechanism of ESI; or 3 ) at least one inaccurate clinical statement about the indications, adverse effects, benefits, and potential outcomes of ESI. The criteria for neither useful nor misleading videos included 1) no scientific information about the definition of ESI; 2) no information on the pain-reducing mechanism of ESI; and 3) no clinical information about the indications, adverse effects, benefits, and potential outcomes of ESI. When a video contained both accurate and inaccurate statements, it was classified as misleading.

Two reviewers (M.C.C and D.P.) assessed the reliability, quality, and usefulness of the included videos, and any discrepancies in assessment were discussed until consensus was reached. The assessment was conducted based on the guidelines for spinel diagnosis and treatment procedures by the Spine Intervention Society. ${ }^{13}$

\section{Statistical Analysis}

The Statistical Package for the Social Sciences 22 (IBM, Armonk, NY, USA) was used for the statistical analysis. The 
Mann-Whitney $U$-test was used to evaluate statistically significant differences in general features of the videos between groups. A statistical significance was set at $\mathrm{p}<0.05$.

\section{Results}

The general features (production source, duration on YouTube, video length, and total number of views, likes, dislikes, and subscribers) of the 50 most-viewed videos are presented in Table 1. The web address and title of videos on YouTube are listed in Supplementary 1. Of these videos, $22 \%(\mathrm{n}=11)$ contained information with high reliability (mDISCERN score: $5, \mathrm{n}=1 ; 4, \mathrm{n}=1 ; 3, \mathrm{n}=$ $9 ; 2, \mathrm{n}=18 ; 1, \mathrm{n}=1 ; 0, \mathrm{n}=20)$. Regarding assessment of information quality, 17 videos (34\%) were of moderate (n $=11,22 \%), \operatorname{good}(\mathrm{n}=2,4 \%)$, or excellent $(\mathrm{n}=4,8 \%)$ quality. Conversely, 44\% $(n=22)$ and $22 \%(n=11)$ were of poor and generally poor quality, respectively. In addition, $76 \%(\mathrm{n}=38)$ were classified as useful videos, and $8 \%(n=4)$ as misleading videos. The other $16 \%(n=8)$ were neither useful nor misleading videos.

Of the 50 videos, 41 were produced by hospitals or physicians, and six videos were made by media organizations. The other three videos were produced by patients who experienced ESI. Eleven videos containing information with high reliability were all produced by hospitals or physicians. Videos produced by media organizations and patients were not found highly reliable. Regarding quality of information, of the 41 videos produced by hospitals or physicians, $34.1 \%(n=14)$ were of moderate $(n=8)$, good

Table I General Features and Video Quality of the Videos

\begin{tabular}{|l|l|}
\hline Video Features & Mean \pm SD (Min-Max) \\
\hline $\begin{array}{l}\text { Duration on YouTube } \\
\text { (months) }\end{array}$ & $77.9 \pm 33.5(24-160)$ \\
\hline Video length (seconds) & $267.2 \pm 197.6(61-1$ I 88$)$ \\
\hline Number of views (n) & $\begin{array}{l}142,133.0 \pm 251,934.9 \\
(1,493,311-3949)\end{array}$ \\
\hline Number of likes (n) & $463.2 \pm 1056.2(1-6800)$ \\
\hline Number of dislikes $(n)$ & $50.3 \pm 85.0(0-431)$ \\
\hline Number of subscribers $(n)$ & $161,083.9 \pm 665,850.1(6-3,460,000)$ \\
\hline mDISCERN score & $\begin{array}{l}1.5 \pm 1.3(0-5)[\geq 3 \text { score, } n=11 \\
(22 \%)]\end{array}$ \\
\hline GQS score & $\begin{array}{l}2.3 \pm 1.1(I-5)[\geq 3 \text { score, } n=17 \\
(34 \%)]\end{array}$ \\
\hline
\end{tabular}

Abbreviations: mDISCERN, modified DISCERN scale; GQS, Global Quality Scale. $(n=2)$, or excellent quality $(n=4)$, and $46.3 \%(n=19)$ and $19.5 \%(\mathrm{n}=8)$ were of generally poor and poor quality, respectively. Of the six videos produced by media organizations, $50 \%(\mathrm{n}=3)$ videos were of moderate quality, and $33.3 \%(n=2)$ and $16.7 \%(n=1)$ were of generally poor and poor quality, respectively. Of three videos produced by patients, 1 was of generally poor quality, and the other two videos were of poor quality. As for usefulness, all 41 videos produced by hospitals or physicians were classified as useful $(82.9 \%, \mathrm{n}=34)$ or neither useful nor misleading $(17.1 \%, \mathrm{n}=7)$. Of the six videos produced by media organizations, four $(66.7 \%)$ were useful, one $(16.7 \%)$ was misleading, and the other one $(16.7 \%)$ was neither useful nor misleading. Misleading videos produced by hospitals, physicians, or media organizations contained exaggerating information regarding the effect of ESI. The three videos made by patients were all assessed as misleading information focusing excessively on the side effect.

The duration on YouTube was significantly longer $(\mathrm{p}=$ $0.006)$ and the number of subscribers was significantly lower $(\mathrm{p}=0.045)$ in highly reliable videos (mDISCERN $\geq 3$ ). The video length and number of views, likes, and dislikes were not significantly different between videos with $\operatorname{mDISCERN} \geq 3$ and those with mDISCERN $<3$ (video length, $\mathrm{p}=0.210$; number of views, $\mathrm{p}=0.615$; number of likes, $\mathrm{p}=0.859$; number of dislikes, $\mathrm{p}=0.780$ ). Videos with moderate to excellent quality (GQS $\geq 3$ ) received fewer likes and subscribers than those with generally poor or poor quality (GQS < 3) (likes, p = 0.047; subscribers, $\mathrm{p}=0.003)$. The other features were not significantly different between videos with GQS $\geq 3$ and those with GQS < 3 (duration on YouTube, $\mathrm{p}=0.064$; video length, $\mathrm{p}=0.085$; number of views, $\mathrm{p}=0.155$; number of dislikes, $\mathrm{p}=0.140$ ). In the comparison between useful videos and misleading or neither useful nor misleading videos, no significant difference was observed (duration on YouTube, $\mathrm{p}=0.496$; video length, $\mathrm{p}=0.176$; number of views, $\mathrm{p}=0.212$; number of likes, $\mathrm{p}=0.468$; number of dislikes, $\mathrm{p}=0.181$; number of subscribers, $\mathrm{p}=0.341$ ).

\section{Discussion}

In this study, we assessed the reliability, quality, usefulness, and general features of the 50 most-viewed YouTube videos on ESI. We found that only $22 \%$ of videos contained information with high reliability, but all these videos were produced by hospitals or physicians. None of the videos produced by media organizations and 
patients were reliable. When hospitals or physicians produce a video on ESI, they should exert more effort to make use of reliable sources of information, provide balanced information, list additional sources of information, and clearly mention areas of uncertainty. Furthermore, especially media organizations and patients need a consultation from specialists on pain procedures or a thorough review of information from reliable sources in order to produce high quality videos on ESI.

As for information quality, only $34 \%$ of the videos were moderate to excellent quality. Even when videos were produced by hospitals or physicians, about half of the videos were generally of poor or poor quality. Video producers should try to improve video quality by providing important information to viewers.

Regarding usefulness of information, although $76 \%$ of videos were assessed to contain useful information, $8 \%(\mathrm{n}=4)$ had misleading information. Of these four videos with misleading information, three were provided by patients who experienced ESI. These videos can lead to misconceptions regarding ESI. When a patient uploads videos on medical information, they should be cautious of the possibility that incorrect or biased information may be included. For the prevention of uploading videos containing misleading information, campaigns or policies from professional societies and governments are needed.

In addition, videos with moderate to excellent quality (GQS $\geq 3$ ) had fewer likes and subscribers than those with generally poor or poor quality. This result indicates that the public had difficulty judging whether information in online videos were well-qualified or not. We think that professional societies related to pain procedures should consider creating and sharing ESI videos on social media. Moreover, our study showed that even hospitals or physicians posted low-reliability and low-quality videos. With the advent and growing importance of social media in the health field, hospitals or physicians should pay special attention to the content of the videos so that reliable and helpful information is disseminated to people. ${ }^{14}$

In conclusion, social media platforms, such as YouTube, are continuing to grow. Through YouTube, the sharing of medical information is spreading. It has become a major source of information for patients deciding whether or not to receive the treatment that clinicians recommended. In the current study, we found the reliability and quality of the most-viewed videos to be low, even those produced by hospitals or physicians.
In addition, there was a tendency toward lower quality when it was produced by media organizations or patients. Furthermore, viewers lacked the ability to judge the quality of information on YouTube. Physicians and professional societies should expend effort in providing balanced and unbiased medication information on ESI, because it is important for patients to make appropriate decisions on their medical treatment. However, our study is limited, in that we did not calculate inter-rater agreement. Further studies compensating this limitation are warranted.

\section{Funding}

This study was supported by National Research Foundation of Korea Grant funded by the Korean government (Grant no. NRF-2021R1A2C1013073).

\section{Disclosure}

The authors report no conflicts of interest in this work.

\section{References}

1. Koes BW, van Tulder MW, Peul WC. Diagnosis and treatment of sciatica. BMJ. 2007;334(7607):1313-1317. doi:10.1136/ bmj.39223.428495.BE

2. Will BE, Synofzik M. Assessing quality of life in patients with lumbar sciatica. Virtual Mentor. 2005;7(2). doi:10.1001/virtualmentor.2005.7.2.cprl1-0502

3. Chang MC. Conservative treatments frequently used for chronic pain patients in clinical practice: a literature review. Cureus. 2020;12(8):e9934.

4. Choo YJ, Chang MC. Effectiveness of orthoses for treatment in patients with spinal pain. Yeungnam Univ J Med. 2020;37(2):84-89. doi:10.12701/yujm.2020.00150

5. Yang S, Boudier-Revéret M, Chang MC. Use of pulsed radiofrequency for the treatment of discogenic back pain: a narrative review. Pain Pract. 2020. doi:10.1111/papr.12978.

6. Do KH, Kim TH, Chang MC. Effects of interlaminar epidural steroid injection in patients with moderate to severe lumbar central spinal stenosis: a prospective study. Ann Palliat Med. 2020;9(2):163-168. doi:10.21037/apm.2020.02.20

7. Lee DG, Ahn SH, Lee J. Comparative effectiveness of pulsed radiofrequency and transforaminal steroid injection for radicular pain due to disc herniation: a prospective randomized trial. J Korean Med Sci. 2016;31:1324-1330. doi:10.3346/jkms.2016.31.8.1324

8. Johansson A, Hao J, Sjölund B. Local corticosteroid application blocks transmission in normal nociceptive C-fibres. Acta Anaesthesiol Scand. 1990;34(5):335-338. doi:10.1111/j.13996576.1990.tb03097.x

9. Ventola CL. Social media and health care professionals: benefits, risks, and best practices. $P$ T. 2014;39(7):491-520.

10. Langford B, Hooten WM, D'Souza S, Moeschler S, D'Souza RS. YouTube as a source of medical information about spinal cord stimulation. Neuromodulation. 2021;24(1):156-161. doi:10.1111/ ner. 13303

11. Charnock D, Shepperd S, Needham G, Gann R. DISCERN: an instrument for judging the quality of written consumer health information on treatment choices. J Epidemiol Community Health. 1999;53(2):105-111. doi:10.1136/jech.53.2.105 
12. Bernard A, Langille M, Hughes S, Rose C, Leddin D, Veldhuyzen van Zanten S. A systematic review of patient inflammatory bowel disease information resources on the World Wide Web. Am $J$ Gastroenterol. 2007;102(9):2070-2077. doi:10.1111/j.15720241.2007.01325.x

13. Bogduk N, ed. Practice Guidelines for Spinal Diagnostic and Treatment Procedures. 2nd ed. San Francisco: International Spine. Intervention Society; 2013.
14. Bornkessel A, Furberg R, Lefebvre RC. Social media: opportunities for quality improvement and lessons for providers-a networked model for patient-centered care through digital engagement. Curr Cardiol Rep. 2014;16(7):504. doi:10.1007/s11886-014-0504-5

\section{Publish your work in this journal}

The Journal of Pain Research is an international, peer reviewed, open access, online journal that welcomes laboratory and clinical findings in the fields of pain research and the prevention and management of pain. Original research, reviews, symposium reports, hypothesis formation and commentaries are all considered for publication. The manuscript

Submit your manuscript here: https://www.dovepress.com/journal-of-pain-research-journa management system is completely online and includes a very quick and fair peer-review system, which is all easy to use. Visit http:// www.dovepress.com/testimonials.php to read real quotes from published authors. 